\title{
Gradenigo Syndrome: Unusual Consequence of Otitis Media
}

\author{
Jennie M. Valles Robert Fekete \\ Department of Neurology, New York Medical College, Valhalla, N.Y., USA
}

\section{Key Words}

Gradenigo syndrome $\cdot$ Otitis media $\cdot$ Mastoiditis $\cdot$ Apical petrositis $\cdot$ Meningitis

\begin{abstract}
Introduction: In 1904, Giuseppe Gradenigo published his case series on the triad of ipsilateral abducens nerve palsy, facial pain in the trigeminal nerve distribution, and suppurative otitis media, which would subsequently be referred to as Gradenigo syndrome. Case Report: Our patient was a 36-year-old female, 23 weeks pregnant, with a 6-day history of right-sided otalgia and hearing loss and a 4-day history of purulent otorrhea, who presented with severe, holocephalic headache, meningeal signs, fever, photophobia, and mental status decline. Lumbar puncture yielded a white blood cell count of 1,559 cells $/ \mathrm{mm}^{3}$ with $95 \%$ polymorphonuclear leukocytes, a red blood cell count of 111 cells $/ \mathrm{mm}^{3}$, a protein level of $61 \mathrm{mg} / \mathrm{dl}$, and a glucose level of $<40 \mathrm{mg} / \mathrm{dl}$. Cerebrospinal fluid Gram stain showed Gram-positive diplococci, which were subsequently identified as Streptococcus pneumoniae and treated with ceftriaxone. On the second hospital day, she developed horizontal diplopia due to right abducens nerve palsy and right mydriasis. Both symptoms resolved on the third hospital day. Erosion of temporal bone and opacification of mastoid air cells was shown on CT scan. A CT venogram showed an irregularity of the left transverse and superior sagittal sinuses. She was treated with enoxaparin for possible sinus thrombosis. Discussion: This case demonstrates rare but serious sequelae of otitis media and Gradenigo syndrome. Holocephalic headache from meningitis masked trigeminal pain. Involvement of the ipsilateral petrous apex and surrounding structures on imaging and clinical improvement with antibiotic treatment supports Gradenigo syndrome over intracranial hypertension due to venous sinus thrombosis as the cause of the abducens nerve palsy.


Valles and Fekete: Gradenigo Syndrome: Unusual Consequence of Otitis Media

\section{Introduction}

In 1904, Giuseppe Gradenigo published his case series on the triad of ipsilateral abducens nerve palsy, facial pain in the trigeminal nerve distribution, and suppurative otitis media, which would subsequently be referred to as Gradenigo syndrome [1, 2]. The syndrome can be associated with serious sequelae including death from meningitis. Primo Dorello reported that inflammatory edema serves to compress the abducens nerve in the canal that was subsequently named after him. The borders of Dorello's canal consist of the petrosphenoidal or Gruber's ligament (posterior/superior), clivus (medial), and anteromedial sphenoid ridge (lateral) [3].

We present a case of suppurative otitis media, meningitis, and abducens nerve palsy, with MRI confirmation of the involvement of the ipsilateral trigeminal nerve, consistent with Gradenigo syndrome. Her case was complicated by possible venous sinus thrombosis.

\section{Case Report}

Our patient was a 36-year-old female, 23 weeks pregnant, who initially presented to an outside urgent care center with right-sided otalgia and hearing loss. She was prescribed a 5day oral course of azithromycin (500 mg on the first day, followed by $250 \mathrm{mg}$ daily). Two days later, she heard a 'pop' in the right ear and developed white followed by brown purulent otorrhea along with conductive hearing loss in the right ear. Five days after the initial presentation, she developed nausea, vomiting, and confusion. She was treated conservatively with intravenous fluids and ondansetron at an outside emergency room and was discharged. On that evening, she became febrile and her mental status worsened to the point that she had difficulty recognizing her husband. The next day, she was taken to another emergency room where she manifested photophobia, phonophobia, nausea, vomiting, neck stiffness, and severe headache. Lumbar puncture was performed, which yielded a white blood cell count of 1,559 cells $/ \mathrm{mm}^{3}$ with $95 \%$ polymorphonuclear leukocytes, a red blood cell count of 111 cells $/ \mathrm{mm}^{3}$, a protein level of $61 \mathrm{mg} / \mathrm{dl}$, and a glucose level of $<40 \mathrm{mg} / \mathrm{dl}$. Cerebrospinal fluid Gram stain showed Gram-positive diplococci, which were subsequently identified as Streptococcus pneumoniae. She was initially treated with vancomycin 1,500 mg i.v. every $12 \mathrm{~h}$ (total 9 doses), ceftriaxone $2 \mathrm{~g}$ i.v. every $12 \mathrm{~h}$, and ampicillin $1 \mathrm{~g}$ i.v. every $4 \mathrm{~h}$ (total 6 doses) and transferred to our hospital for further evaluation. She was continued on a course of ceftriaxone $2 \mathrm{~g}$ i.v. every $12 \mathrm{~h}$ for a total of 2 weeks followed by oral cefuroxime $500 \mathrm{mg}$ p.o. every $12 \mathrm{~h}$ for an additional 2 weeks.

On the second hospital day in our institution, she developed horizontal diplopia due to abducens nerve palsy as well as mydriasis on the right. Myringotomy was performed by an otolaryngology consultant. She began to improve clinically on the third hospital day with near resolution of photophobia and nausea. Abducens nerve palsy and mydriasis resolved, but conductive hearing loss and mild right-sided headache persisted during the fifth hospital day.

CT temporal bone imaging revealed erosion of the temporal bone (fig. 1). Right mastoid air cell opacification and involvement of the petrous apex is demonstrated on noncontrast MRI brain (fig. 2).

She was put on enoxaparin injection $80 \mathrm{mg}$ s.c. twice a day for treatment of venous sinus thrombosis given narrowing and contour irregularity of the left transverse sinus as well as the posterior and distal aspect of the superior sagittal sinus on CT venography with contrast. 
Valles and Fekete: Gradenigo Syndrome: Unusual Consequence of Otitis Media

MR venography with contrast could not be performed due to concerns about teratogenesis from gadolinium.

\section{Discussion}

S. pneumoniae is a common pathogen implicated in both otitis media and meningitis. Given temporal bone erosion in figure 1, it is likely that meningitis was initially caused by petrositis. Her symptoms of diplopia and otorrhea are consistent with Gradenigo syndrome. Ipsilateral facial pain was masked by a holocephalic headache from meningitis and possible venous sinus thrombosis. Ipsilateral trigeminal nerve hyperintensity on T2-weighted MRI confirmed this nerve's involvement (fig. 3).

In the modern antibiotic era, Gradenigo syndrome is exceedingly rare. It has been found in association with cholesteatoma, but there was no suggestion of cholesteatoma on imaging or examination [4].

An alternative explanation for the patient's symptoms would be infectious venous sinus thrombosis with a resulting increase in intracranial pressure leading to mydriasis and abducens nerve palsy. Infection, in this case from mastoiditis, and pregnancy are risk factors for venous sinus thrombosis [5]. Involvement of the ipsilateral petrous apex and surrounding structures on imaging and clinical improvement with antibiotic treatment supports Gradenigo syndrome over intracranial hypertension due to venous sinus thrombosis as the cause of the abducens nerve palsy.

Oral azithromycin course treatment failure in this case most likely occurred due to macrolide resistance [6]. Given that mastoiditis/petrositis can lead to grave complications of meningitis, cranial nerve involvement, and venous sinus thrombosis, early recognition and appropriate change in therapy in case of resistance is crucial.

\section{References}

1 Gradenigo G: Über die Paralyse des Nervus abducens bei Otitis. Arch Ohrenheilkunde 1907;774:149-187.

-2 Matis GK, de A Silva DO, Chrysou OI, Karanikas MA, Birbilis TA: Giuseppe Gradenigo: much more than a syndrome! Historical vignette. Surg Neurol Int 2012;3:122.

-3 Ezer H, Banerjee AD, Thakur JD, Nanda A: Dorello's canal for laymen: a Lego-like presentation. J Neurol Surg B Skull Base 2012;73:183-189.

-4 Zengel P, Wiekström M, Jäger L, Matthias C: Isolated apical petrositis: an atypical case of Gradenigo's syndrome. HNO 2007;55:206-210.

5 Skeik N, Stark MM, Tubman M: Complicated cerebral venous sinus thrombosis with intracranial hemorrhage and mastoiditis. Vasc Endovascular Surg 2012;46:585-590.

6 Jenkins SG, Farrell DJ: Increase in pneumococcus macrolide resistance, United States. Emerg Infect Dis 2009;15:1260-1264. 


\section{Case Reports in Neurology}

\begin{tabular}{l|l}
\hline Case Rep Neurol 2014;6:197-201 \\
\hline DOI: 10.1159/000365843 & $\begin{array}{l}\text { ○ 2014 S. Karger AG, Basel } \\
\text { www.karger.com/crn }\end{array}$ \\
\hline
\end{tabular}

Valles and Fekete: Gradenigo Syndrome: Unusual Consequence of Otitis Media

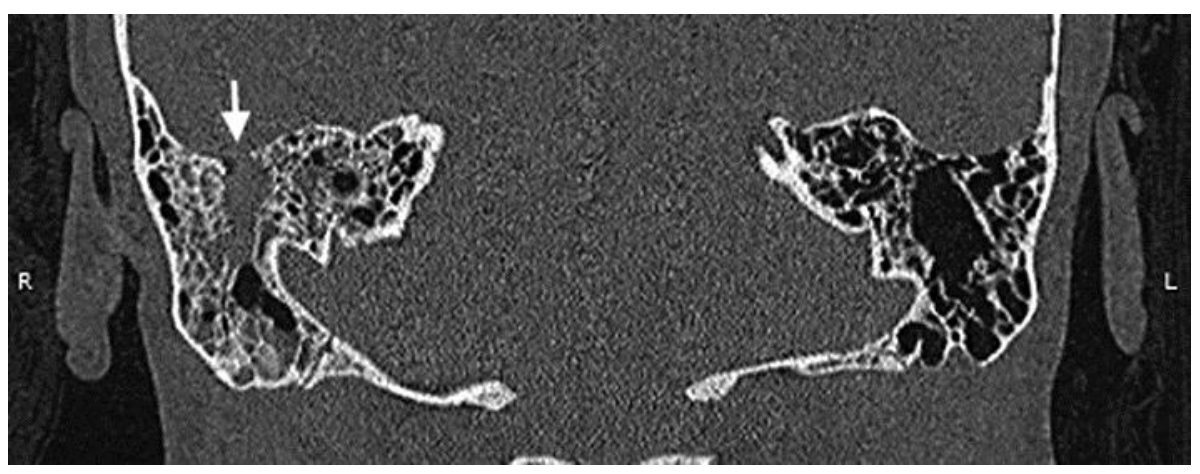

Fig. 1. Noncontrast CT scan showing temporal bone erosion (arrow) and right mastoid opacification.

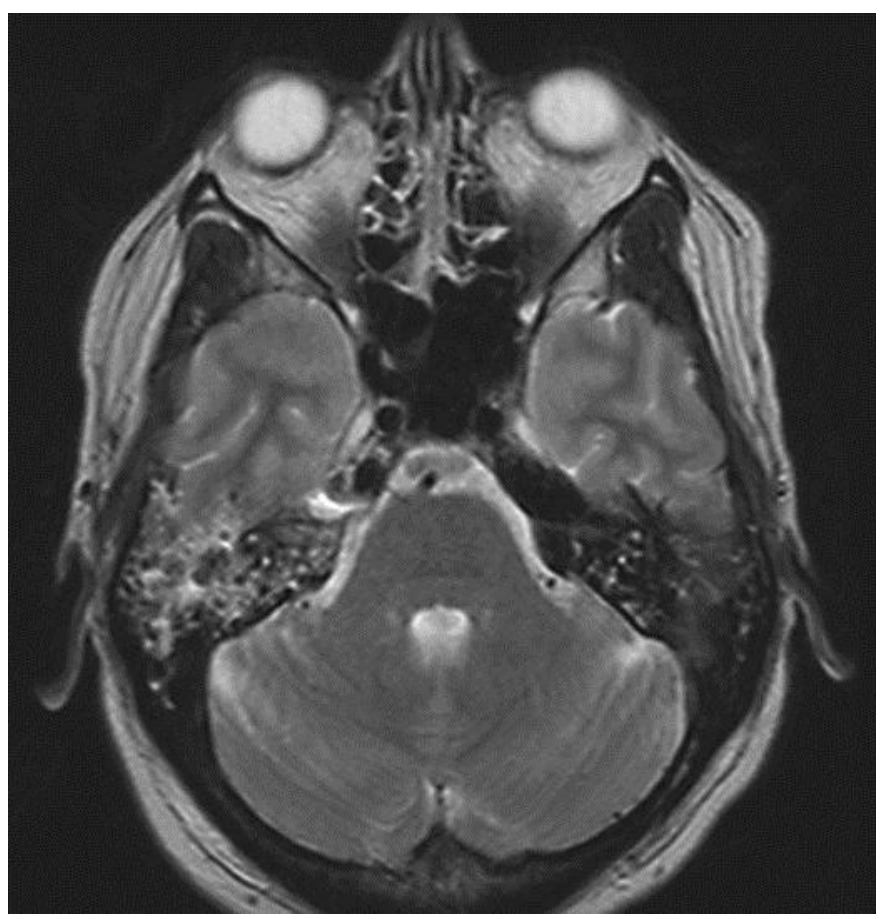

Fig. 2. Noncontrast T2-weighted MRI demonstrating right mastoid opacification. 
Valles and Fekete: Gradenigo Syndrome: Unusual Consequence of Otitis Media

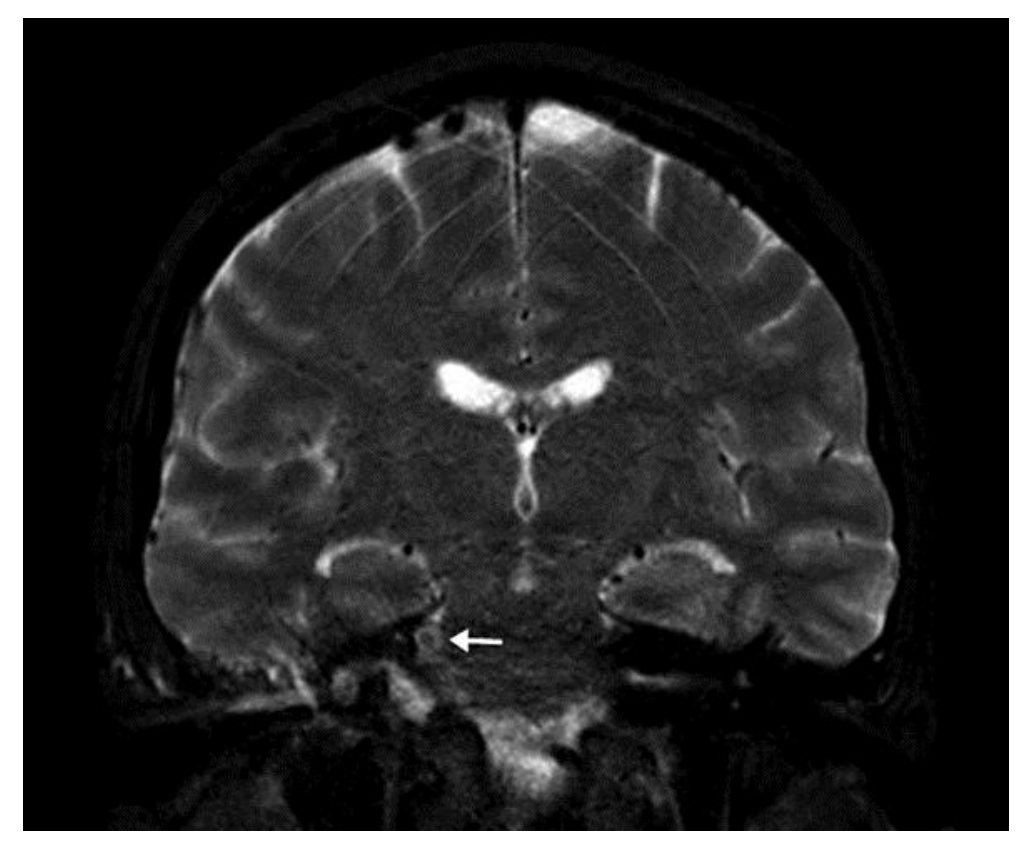

Fig. 3. Noncontrast T2-weighted MRI showing hyperintensity of the right trigeminal nerve (arrow). 